\title{
An axion-induced SM/MSSM Higgs landscape and the Weak Gravity Conjecture
}

\author{
Alvaro Herráez and Luis E. Ibáñez \\ Departamento de Física Teórica and Instituto de Física Teórica UAM/CSIC, \\ Universidad Autónoma de Madrid, \\ Cantoblanco, 28049 Madrid, Spain \\ E-mail: alvaro.herraez@uam.es, luis.ibannez@uam.es
}

ABSTRACT: We construct models in which the SM Higgs mass scans in a landscape. This is achieved by coupling the SM to a monodromy axion field through Minkowski 3-forms. The Higgs mass scans with steps given by $\delta m_{H}^{2} \simeq \eta \mu f$, where $\mu$ and $f$ are the axion mass and periodicity respectively, and $\eta$ measures the coupling of the Higgs to the associated 3 -form. The observed Higgs mass scale could then be selected on anthropic grounds. The monodromy axion may have a mass $\mu$ in a very wide range depending on the value of $\eta$, and the axion periodity $f$. For $\eta \simeq 1$ and $f \simeq 10^{10} \mathrm{GeV}$, one has $10^{-3} \mathrm{eV} \lesssim \mu \lesssim 10^{3} \mathrm{eV}$, but ultralight axions with e.g. $\mu \simeq 10^{-17} \mathrm{eV}$ are also possible. In a different realization we consider landscape models coupled to the MSSM. In the context of SUSY, 4-forms appear as being part of the auxiliary fields of SUSY multiplets. The scanning in the 4-forms thus translate into a landscape of vevs for the $N=1$ auxiliary fields and hence as a landscape for the soft terms. This could provide a rationale for the MSSM fine-tuning suggested by LHC data. In all these models there are 3 -forms coupling to membranes which induce transitions between different vacua through bubble nucleation. The Weak Gravity Conjecture (WGC) set limits on the tension of these membranes and implies new physics thresholds well below the Planck scale. More generaly, we argue that in the case of string SUSY vacua in which the Goldstino multiplet contains a monodromy axion the WGC suggests a lower bound on the SUSY breaking scale $m_{3 / 2} \gtrsim M_{s}^{2} / M_{p}$.

Keywords: Beyond Standard Model, Effective field theories, Higgs Physics, Supersymmetric Effective Theories

ARXIV EPRINT: 1610.08836 


\section{Contents}

1 Introduction 1

2 Axions and 3-forms 4

3 A Higgs landscape from axion monodromy. A minimal model 5

3.1 Stability and the Weak Gravity Conjecture 8

3.2 The Hierarxion 9

4 A MSSM landscape $\quad 11$

5 The SUSY breaking scale, the string scale and the Weak Gravity Con$\begin{array}{ll}\text { jecture } & 14\end{array}$

$\begin{array}{lll}6 & \text { Comments and conclusions } & 17\end{array}$

\section{Introduction}

There are a couple of very bizarre small mass scales in physics. One is the cosmological constant which, if identified with dark energy, is of order $V_{0} \simeq\left(10^{-3} \mathrm{eV}\right)^{4}$, ridiculously small compared to any other scale in the theory. The other is the Electro-Weak(EW) scale which is of order $m_{H} \simeq 10^{2} \mathrm{GeV}$, much smaller than any expected ultraviolet(UV) cut-off. Possibly the best solution to the first question was suggested by Weinberg [1], who pointed out thar if the c.c. $V_{0}$ scans in a large multiplicity of finely-grained values, galaxy formation requires $V_{0}$ to be positive and of order the presently observed values. This is a remarkable prediction, since it was pointed out before the existence of dark energy was confirmed.

A natural question is whether an analogous mechanism could be at work for the Higgs hierarchy problem. The EW scale is tied up to the mass parameter $m_{H}^{2}$ of the Higgs boson, which is unstable under radiative corrections and would be expected to be of order the cutoff scale $m_{H}^{2} \simeq \Lambda_{\mathrm{UV}}^{2}$. One way to stabilize the Higgs mass is low energy SUSY. However the observed relatively large Higgs mass suggests that SUSY, if present, is possibly beyond the reach of LHC or much heavier. So, even though SUSY still remains the most ellegant solution to the hierarchy problem it makes sense to look for alternative or complementary solutions.

In the present paper we study the generation of a landscape of Higgs mass parameters $m_{H}^{2}$ to address the EW hierarchy problem. This landscape will contain a large number of possible values for $m_{H}^{2}$ from large and negative (or positive) to small with $m_{H}^{2}$ in the observed phenomenological range. For the observed value of the EW scale to be one of the possibilities in the landscape, we need $m_{H}^{2}$ to scan with a fine-grain mass scale a fraction of the EW mass scale $m_{W}$. In fact anthropic considerations require the EW vev not to 


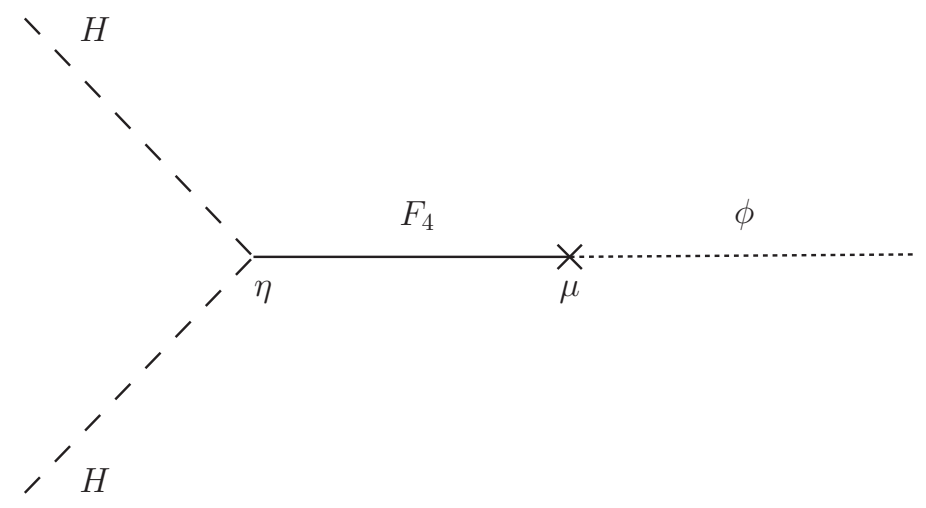

Figure 1. Scheme of the Higgs-axion system. As the axion completes one cycle as $\phi \rightarrow \phi+f$, the Higgs mass $^{2}$ changes by $\delta\left(m_{H}^{2}\right) \simeq \eta \mu f$.

be far from the measured value $v_{0}=170 \mathrm{GeV}$. Defining $v=v_{0}+\delta v$ one finds constraints (see [2] for the asumptions used in this estimate)

$$
0.39 \leq \frac{\left|v_{0}+\delta v\right|}{v_{0}} \leq 1.64
$$

which implies

$$
\frac{\delta m_{H}^{2}}{m_{H_{0}}^{2}}=2 \frac{\delta v}{v_{0}} \leq 1.2
$$

These limits come essentially from the atomic principle, i.e. imposing that complex and stable nuclei can form. Note that it requires $\delta v \leq 0.6 v_{0}$ and hence practically determine the weak scale to be what experimentally is. These constrains may be considered a necessary but not a sufficient condition for an anthropic solution to the hierarchy problem. Indeed, it is well known that the masses of the first generation quarks and leptons would also need to scan in an anthropic setting, see e.g. [2-6]. In this paper we will only address the issue of a landscape of Higgs mass parameters which is necessary for an antropic solution to work. Note in this connexion that we we will not try to look here for Higgs mass distributions which are peaked around the EW scale. For an anthropic solution of the hierarchy problem it is enough to show that there is a landscape of Higgss masses which contains the observed Higgs mass, it does not need to be the most likely value. The purpose of this paper is to construct models in which indeed the Higgss mass scans and hence completes the above atomic principle into a possible solution to the hierarchy problem. For a discussion of some phenomenological scenarios from a field theory landscape see also [7].

We consider two classes of models, non-SUSY and SUSY, with some important differences between them. In both cases the important ingredient is the existence of Minkowski 3 -forms $C_{3},{ }^{1}$ which couple to the Higgs sector via their field strength $F_{4}$. These 4 -forms in turn couple to an axion-like field $\phi$ in such a way that a built-in shift symmetry under $\phi \rightarrow \phi+f$ is respected. The 4 -forms are quantized in units of $\mu f$, with $\mu$ the axion mass.

\footnotetext{
${ }^{1}$ For pioneer works involving 3-forms see e.g. [8-16]. For more recent applications see [17-22, 24, 25]. Four-form-Higgs couplings were considered by Dvali in [17-20].
} 
In the simplest non-SUSY example the 4-form couples to the Higgs in a renormalizable way, with a term $\eta F_{4}|H|^{2}$ (see figure (1)). This coupling makes the Higgs mass to scan in a landscape of values in steps given by $\eta \mu f \lesssim m_{W}^{2}$. The mass of this axion-like field (or Hierarxion) is hence of order $\mu \lesssim m_{W}^{2} / f$, which is tipically very small.

In the SUSY case, the 4-forms are part of the auxiliary field system of the $N=1$ multiplets. The coupling of the Higgs system to the 4-forms appear as in standard gravity mediation, so the Higgs fields get mass ${ }^{2}$ of order $F_{4}^{2} / M_{p}^{2}$. This suggests to identify the vev of $F_{4}$ with an intermediate scale, $F_{4} \simeq\left(10^{10}\right)^{2} \mathrm{GeV}^{2}$, so that one obtains Higgs masses of order the EW scale. Within string theory the 3 -forms associated to these 4 -forms couple to membranes whose tension would be typically of order the string scale, i.e. $\left(F_{4}^{3 / 2}\right) \simeq M_{s}^{3}$. Thus the string scale will typically be of order the intermediate scale, $M_{s} \simeq 10^{10} \mathrm{GeV}$. Again, the fact that the auxiliary fields related to the 4-forms are quantized, makes the Higgs mass and in general all soft terms to scan in a landscape of values around the EW scale. In particular, the possibility exists that soft terms could be relatively large compared to LHC scales, say 3-5 TeV and still having correct fine-tuned EW breaking by a fortuitous cancellation of different 4-form contributions in the landscape.

The 3-forms couple to membranes which may nucleate transitions in these landscapes of vacua. The Weak Gravity Conjecture (WGC) $[26-28]^{2}$ strongly constraints the tension of the membranes involved, as well as the mass of the axion. In the non-SUSY examples the membrane tension is bounded above by $T \lesssim \eta^{-1}\left(10^{8}\right)^{3} \mathrm{GeV}^{3}$, with $\eta$ the 4-form-Higgs coupling. The corresponding axion is bounded below as $\mu_{\text {axion }} \gtrsim T / 2 \pi f M_{p}$, and impossing further stability against nucleation one has $\mu_{\text {axion }} \gtrsim 10^{-3} \mathrm{eV}\left(10^{10} \mathrm{GeV} / f\right)\left(m / 10^{10} \mathrm{GeV}\right)^{3 / 2}$, with $m$ the UV Higgs cut-off. In the SUSY examples the WGC suggests that $m_{3 / 2} \gtrsim$ $T / f M_{p}$. Within string theory one expects $T^{1 / 3} \simeq f \simeq M_{s}$, with $M_{s}$ the string scale, so that one gets $m_{3 / 2} \gtrsim M_{s}^{2} / M_{p}$. This implies that if we want to have SUSY breaking soft terms of order the EW scale, the string scale should be of order the intermediate scale $M_{s} \simeq \sqrt{M_{W} M_{p}} \simeq 10^{10} \mathrm{GeV}$ or below. This is a very strong constraint, since the typical scenario with $M_{s} \simeq 10^{16} \mathrm{GeV}$ would be ruled out in this context.

In the non-SUSY version of this idea there is a small scale given by $\eta q \leq M_{W}$, where $q$ is the charge of the membranes and $\eta$ the Higgs-4-form coupling. The size of the electroweak scale is fixed by a combination of anthropic constraints and the fact that the Higgs vev scans with steps $\eta q$ smaller than $M_{W}$. This implies that we are assuming the existence of a new effective small scale $\eta q \ll M_{p}$. Still this is in principle no worse than e.g. the addition of a $\mu$-term in the MSSM solution to the hierarchy problem. On the other hand, in the SUSY version of the present mechanism the size of the EW scale is fixed by the geometric ratio $F_{4} / M_{p}$, which is of the right order if the SUSY breaking scale is at the intermediate scale. In this sense the combination of SUSY and landscape ideas would seem to fit nicely together.

More generally, we argue that in certain classes of string compactifications in which SUSY is broken by fluxes, and the Goldstino multiplet contains a monodromy axion, the

\footnotetext{
${ }^{2}$ See e.g. $[24,29-49]$ for recent WGC papers.
} 
Weak Gravity Conjecture suggests a lower bound on the SUSY breaking scale $m_{3 / 2}$

$$
m_{3 / 2} \geq \frac{M_{s}^{2}}{M_{p}}
$$

This bound has a number of loopholes, some of which are discussed in the text. Still, if true, it would have important phenomenological implications. For example, having low energy SUSY at $1 \mathrm{TeV}$ would require a string scale at $10^{10} \mathrm{GeV}$.

The structure of the rest of this paper is as follows. In the next section we review a few facts about Minkowski 3-forms and their interaction with axions. In section 3 we construct a minimal (non-SUSY) model in which a Higgs mass landscape is generated in terms of quantized shifts of an axion. We also study the instability of the model against buble nucleation and constraints on the axion mass and scale of new physics from the WGC. Limits on the mass of these axions are given. In section four we address the construction of $N=1$ SUSY models with a Higgs mass landscape. We discuss the mentioned lower bound on the SUSY breaking scale from the WGC in section five and leave the last section for some general comments and conclussions.

\section{Axions and 3-forms}

Before presenting the model let us briefly review a few facts about these Minkowski 3 -forms (see e.g. $[13-23,50-52]$ ). The action for a 3 -form $C_{\nu \rho \sigma}$ is given by

$$
\mathcal{L}=-\frac{1}{2} F_{\mu \nu \rho \sigma} F^{\mu \nu \rho \sigma}+S_{\text {bound }}
$$

where $F=d C$ is the field-strength 4 -form and $\mathcal{L}_{\text {bound }}$ includes some boundary terms which, although necessary to get the right field equations (see e.g. the discussion in [21, 52]), will not be relevant in our discussion. The equations of motion imply that the 4 -form is a constant tensor in Minkowski,

$$
F_{\mu \nu \rho \sigma}=f_{0} \epsilon_{\mu \nu \rho \sigma}
$$

where $f_{0}$ is a real constant of mass dimension two. Note that $f_{0}$ behaves as a constant electric 4-form field permeating the whole Minkoski space and contributing (positively) to the vacuum energy in a way proportional to $f_{0}^{2}$. We see that a 3 -form has no propagating degrees of freedom. Still it may have interesting dynamics. In particular, 3-forms naturally couple to the worldvolume of membranes (or domain walls) through

$$
S_{\mathrm{mem}}=q \int_{D_{3}} d^{3} \xi \epsilon^{a b c} C_{\mu \nu \rho}\left(\frac{\partial X^{\mu}}{\partial \xi^{a}} \frac{\partial X^{\nu}}{\partial \xi^{b}} \frac{\partial X^{\rho}}{\partial \xi^{c}}\right)
$$

where the membrane charge $q$ has dimensions of mass $^{2}$ and $D_{3}$ is the membrane world volume. Due to this coupling, regions of space separated by membranes change their 4 -form background $f_{0}$ by

$$
f_{0} \longrightarrow f_{0}+n q, \quad n \in \mathbf{Z} .
$$


So membrane nucleation yield changes in the value of the 4-form background which are quantized in units of the membrane charge. At this level the value of the charge $q$ is undetermined. However there is an interesting way in which the value of $q$ turns out to be constrained. This happens if the 3 -form is coupled to an axion-like scalar giving it a mass. Let us introduce an axion-like field $\phi$, i.e., a pseudoscalar with a discrete shift symmetry under

$$
\phi \longrightarrow \phi+m f, \quad m \in \mathbf{Z},
$$

with $f$ the axion periodicity. Let us consider the addition to the action of a direct coupling of the axion to the 4 -form

$$
S=-\frac{1}{2}\left(\partial_{\mu} \phi\right)^{2}-\frac{1}{2} F^{2}+\mu \phi F .
$$

Using the equations of motion for $F$ one obtains a scalar potential

$$
V=\frac{1}{2}\left|f_{0}+\mu \phi\right|^{2}
$$

where we have allowed for a 4 -form vev $f_{0}$. Note that, even though now the axion has mass $\mu$, the axion shift symmetry is respected if the 4-form also transforms apropriately

$$
\phi \rightarrow \phi+n f, \quad f_{0} \rightarrow f_{0}-n \mu f .
$$

Comparing eqs. (2.4) and (2.8) one obtains the consistency condition for the charge $q$ of membranes coupling to this axion system

$$
p q=\mu f, \quad p \in \mathbf{Z} .
$$

This equation relates the otherwise undetermined membrane charge $q$ to the axion parameter product $\mu f$. This constraint will be interesting below, when we construct a specific model couple to the Higgs. In what follows we will assume take $|q|=\mu f$ as the natural value for the 4 -form quanta and briefly discuss the more general case below. This process in which the axion gets mass may be understood as a generalized Higgs mechanism in which the 2 -form $B_{\rho \sigma}$ dual to the axion field is swallowed and gains a mass $\mu$. Indeed after this duality the mass term becomes

$$
-\frac{\mu^{2}}{2}\left|C_{3}-d B_{2}\right|^{2}
$$

which indeed realizes a generalized Higgs mechanism.

Let us close this section by noting that Minkowski four-forms appear naturally in string theory upon reduction to four dimensions of higher dimensional RR and NS antisymmetric fields, see e.g. $[15,16,21,23,25,50,51]$.

\section{A Higgs landscape from axion monodromy. A minimal model}

Let us now couple this axion/3-form system to the SM Higgs field $H$. For reasons to be obvious later the minimal model one can build involves two 4 -forms $F_{a}$ and $F_{h}$. The 
latter is by definition the linear combination of the two 4-forms which couples to the Higgs through a $\operatorname{dim}=4$ operator. The relevant piece of the action is then ${ }^{3}$

$$
\mathcal{L}=-\frac{1}{2}\left(F_{a}\right)^{2}-\frac{1}{2}\left(F_{h}\right)^{2}+\phi\left(\mu F_{a}+\mu^{h} F_{h}\right)+\eta F_{h}|H|^{2} .
$$

Here $\eta$ is an adimensional coupling constant. Using the equations of motion for the 4-forms one finds the potential

$$
V=\frac{1}{2}\left|f_{0}^{a}+\mu \phi\right|^{2}+\frac{1}{2}\left|f_{0}^{h}+\mu^{h} \phi+\eta \sigma^{2}\right|^{2}
$$

where we have set the Higgs to its physical neutral component $|H|^{2}=\sigma^{2}$. Note that this scalar potential is invariant under the axion shift symmetry

$$
\phi \rightarrow \phi+n f ; \quad f_{0}^{a} \rightarrow f_{0}^{a}-\mu n f ; \quad f_{0}^{h} \rightarrow f_{0}^{h}-\mu^{h} n f, n \in \mathbf{Z} .
$$

The membranes coupling to these 3 -forms will have charges $q_{a}, q_{h}$ related to the axion parameters as

$$
q_{a}=\mu f, \quad q_{h}=\mu^{h} f .
$$

The above shift symmetry guarantees that the mass parameters $\mu, \mu_{h}$ are stable under loop corrections, the form of the axion dependent potential above will remain even after these corrections. On the other hand the Higgs field couples to the full SM through gauge and Yukawa interactions which will induce masses and quartic coupling corrections. Thus the scalar potential will have really the form

$$
V=\frac{1}{2}\left|f_{0}^{a}+\mu \phi\right|^{2}+\frac{1}{2}\left|f_{0}^{h}+\mu^{h} \phi+\eta \sigma^{2}\right|^{2}-m^{2} \sigma^{2}+\lambda \sigma^{4}
$$

once corrections are taken into account. Here $m^{2}$ will typically be of order the UV scale, since the Higgss mass is unprotected. The minimization conditions require

$$
\begin{aligned}
& \partial V / \partial \sigma=\sigma\left[2 \eta\left(f_{0}^{h}+\mu^{h} \phi+\eta \sigma^{2}\right)+4 \lambda \sigma^{2}-2 m^{2}\right]=0 \\
& \partial V / \partial \phi=\mu\left(f_{0}^{a}+\mu \phi\right)+\mu^{h}\left(f_{0}^{h}+\mu^{h} \phi+\eta \sigma^{2}\right)=0 .
\end{aligned}
$$

One then finds

$$
\phi=-\frac{f_{0}^{a} \mu+f_{0}^{h} \mu^{h}+\eta \mu^{h} \sigma^{2}}{\mu^{2}+\left(\mu^{h}\right)^{2}},
$$

with the Higgs vev given by

$$
\sigma^{2}=\frac{m^{2}-\eta\left(\cos ^{2} \theta f_{0}^{h}-\sin \theta \cos \theta f_{0}^{a}\right)}{2 \lambda+\eta^{2} \cos ^{2} \theta}
$$

where

$$
\sin ^{2} \theta=\frac{\left(\mu^{h}\right)^{2}}{\mu^{2}+\left(\mu^{h}\right)^{2}}, \quad \cos ^{2} \theta=\frac{\mu^{2}}{\mu^{2}+\left(\mu^{h}\right)^{2}} .
$$

\footnotetext{
${ }^{3}$ Note that this is a two 4-form generalization of the relaxion model constructed in section (3.2) of [24]. However here we are not considering a relaxion type of model [53] and cosmology plays no crucial role in this landscape construction.
} 
The mass squared matrix of both scalars has the form

$$
M^{2}=\left(\begin{array}{cc}
\mu^{2}+\left(\mu^{h}\right)^{2} & 2 \eta \mu^{h} \sigma_{\min } \\
2 \eta \mu^{h} \sigma_{\min } & 2 \mathcal{M}_{\sigma \sigma}^{2}
\end{array}\right)
$$

with

$$
\mathcal{M}_{\sigma \sigma}^{2}=\frac{4 \lambda+2 \eta^{2}}{2 \lambda+\eta^{2} \cos ^{2} \theta}\left(m^{2}-\eta\left(f_{0}^{h} \cos ^{2} \theta-f_{0}^{a} \sin \theta \cos \theta\right)\right)
$$

The Higgs vev at the minimum can also be written in terms of $\mathcal{M}_{\sigma \sigma}$, evaluated at the minimum

$$
\sigma^{2}=\frac{M_{\sigma \sigma}^{2}}{4 \lambda+2 \eta^{2}}
$$

Looking at eq. (3.12) and (3.13) we see that the Higgs vev scans in a landscape as we vary the 4 -form vevs $f_{0}^{h}, f_{0}^{a}$. There are always potentials in which the Higgs vev obeys eq. (1.1) as long as the step of the 4 -forms $q_{h}, q_{a}$ are of order the observed $M_{H}^{2}$ or smaller. In particular if we change the 4 forms by an amount

$$
f_{0}^{h} \rightarrow f_{0}^{h}+m_{h} q_{h}, \quad f_{0}^{a} \rightarrow f_{0}^{a}+m_{a} q_{a}, \quad m_{h}, m_{a} \in \mathbf{Z}
$$

then the Higgs vev changes by the amount

$$
\delta\left(\sigma^{2}\right)=\mu f \frac{\eta \sin \theta \cos \theta}{\left(2 \lambda+\eta^{2} \cos ^{2} \theta\right)}\left(m_{a}-m_{h}\right)
$$

or, alternatively, in terms of the 4-form quanta via eq. (3.4)

$$
\delta\left(\sigma^{2}\right)=\frac{\eta \mu f}{\left(2 \lambda+\eta^{2} \cos ^{2} \theta\right)} \frac{q_{a} q_{h}}{q_{a}^{2}+q_{h}^{2}}\left(m_{a}-m_{h}\right) .
$$

Note that if there is no coupling of the Higgs to the axion $(\eta=0)$ there is obviously no possibility of fine-tuning. Also the two 4 -forms are required to couple to the axion so that both $q_{a}, q_{h} \neq 0$. Assuming both masses $\mu, \mu^{h}$ to be of the same order (in order not to introduce further hierarchies), which also implies 4-form quanta of the same order one can obtain a fine-tuning as small as required by imposing

$$
\delta\left(\sigma^{2}\right) \simeq \eta \mu f=\eta q_{a} \leq M_{H^{0}}^{2}
$$

So the fine-tuning is directly connected to the 4 -form quanta $q_{a}, q_{h}$ and to the strength of the coupling of the 4-form to the Higgs. We thus have a large family of SM vacua with different Higgs masses, including a number of them consistent with what is observed. Note however that the 4 -form values $f_{0}^{a}, f_{0}^{h}$ themselves are very large, of order the Higgs cut-off mass $m^{2}$, whereas the membrane charges $q_{a}, q_{h}$ are of order the EW scale. This is unlike the SUSY scenario discussed below, in which both are typically of the same order. 


\subsection{Stability and the Weak Gravity Conjecture}

Given the large multiplicity of Higgs vacua, an interesting question is the stability of these against membrane nucleation. If these vacua where very short-lived, the solution to the hierarchy problem would be gone. We can make an estimation using the Coleman-De Lucia computation [54] of the transition rate in the thin wall approximation. The rate is proportional to

$$
P \simeq e^{-B}, \quad B=\frac{27 \pi^{2} T^{4}}{2(\Delta V)^{3}}
$$

where $T$ is the tension of the bubbles (membranes) which can nucleate. We can estimate $\Delta V$, which is the change in the vacuum energy induced by a change $f_{0} \rightarrow f_{0}+q$ in one of the 4 -forms, as

$$
\Delta V \simeq q f_{0} \simeq \frac{q}{\eta} m^{2},
$$

where $m^{2}$ is of order the Higgs cut-off scale, since the 4 -form vevs have to cancel a large quadratically divergent Higgs mass. On the other hand actually we do not know what the tension of the membranes $T$ is. In any event, from $B>1$, in order to have a supresed rate, assuming that for fine-tuning one also requires $\eta q \simeq m_{H}^{2}$, the tension will be bounded below by

$$
T \gtrsim 0.3 \times \eta^{-3 / 2}\left(m_{H}^{2} m^{2}\right)^{3 / 4} .
$$

Note that if the associated membranes are fundamental, like e.g. D2-branes in String Theory, a tension $T=(M)^{3}$ implies the existence of a new physics scale $M$, like the string scale in the case of String Theory. So the above arguments would give a lower bound on such a scale, depending on the size of the Higgs-4-form coupling. Thus one has from stability against nucleation

$$
M^{2} \gtrsim 0.5 \eta^{-1} m_{H} m
$$

where $m$ is the UV cut-off of the Higgs mass. Thus for e.g. $\eta \simeq 1$ one has $M>\sqrt{m_{H} m} \simeq$ $10^{9} \mathrm{GeV}$ if $m \simeq 10^{16} \mathrm{GeV}$.

To gain further insight into the mass scales involved we can try to impose further consistency conditions. In particular it has been argued that the Weak Gravity Conjecture extended to 3 -forms give us an upper bound on the tension $T$ of membranes coupling to 3 -forms. One has [24]

$$
T \leq 2 \pi q M_{p},
$$

where in our case the membrane charge is given by $q=\mu f$, so that we get

$$
T \leq 2 \pi \mu f M_{p} \leq 2 \pi \frac{M_{H}^{2}}{\eta} M_{p} \simeq \frac{1}{\eta}\left(10^{8} \mathrm{GeV}\right)^{3} .
$$

where again we are assuming here $\eta \mu f \simeq m_{H}^{2}$. Note that by making the coupling small, the tension of the membranes can be made large. For example one could have $T \simeq\left(10^{16} \mathrm{GeV}\right)^{3}$ if $\eta \simeq 10^{-24}$. However if e.g. $\eta \simeq 1$ a threshold of new physics should appear around or below $10^{8} \mathrm{GeV}$. 
Combining equations (3.20) and (3.23) one obtains an upper bound on the UV cut-off coming from imposing supresed nucleation and the WGC constraint given by

$$
m \lesssim 7.6 \times \eta^{1 / 3}\left(M_{H} M_{p}^{2}\right)^{1 / 3} \simeq \eta^{1 / 3} 10^{14} \mathrm{GeV} .
$$

Then in this scheme scalar cut-offs $m$ as large as $10^{14} \mathrm{GeV}$ can be fine-tuned in a manner consistent with both the Weak Gravity Conjecture and stability against nucleation. However this scale $m$ is reduced if the coupling $\eta$ is reduced.

Let us make a couple of comments about possible slight modifications to the above results.

1) We have imposed a very conservative upper bound on the value of the Higgs mass fine tuning, $\delta m_{H}^{2} / m_{H}^{2} \leq 1$. We can equally consider a more finely grained fine-tuning

$$
\delta m_{H}^{2} \leq \xi m_{H}^{2}
$$

with $\xi$ as small as we wish. Then the scales and bounds estimated in eqs. (3.20), (3.23) and (3.24) remain applicable replacing in those equations $m_{H}^{2} \rightarrow \xi m_{H}^{2}$. In particular the lower bound on the membrane tension from stability becomes weaker whereas the upper bounds on the tension coming from the WGC becomes stronger, and so happens with the UV scale $m$. The dependence on $\xi$ is however weak, due to the $1 / 3$ power.

2) In the above estimations we consider the quantization constraints $q_{a}=\mu f, q_{h}=\mu^{h} f$. One can equally consider the more general case in which the membrane quanta are integer fractions of the axion shift, as in eq. (2.9). All the results above still apply replacing $q_{a} \rightarrow n_{a} q_{a}, q_{h} \rightarrow n_{h} q_{h}$, with $n_{a}, n_{h} \in \mathbf{Z}$.

Note that one can also obtain a finer tuning (at fixed $\mu f$ ) by reducing the value of the coupling $\eta$ and playing around with the integers $n_{a}, n_{h}$ just mentioned.

\subsection{The Hierarxion}

One interesting feature of this approach is that there is a new particle, me may call it the Hierarxion, which could perhaps have testable properties depending on the masses $\mu$, periodicity $f$ and the possible presence of additional couplings to other SM fields beyond the Higgs like e.g. photons. Concerning the mass of the axion we approximately have

$$
m_{\text {axion }}^{2}=\mu^{2}+\left(\mu^{h}\right)^{2}=\frac{q_{a}^{2}+q_{h}^{2}}{f^{2}} \lesssim \frac{M_{H}^{4}}{\eta^{2} f^{2}}
$$

where the latter inequality comes from the fine-tuning condition, assuming $\xi \simeq 1$. There is also a lower bound on the axion mass if one applies the WGC argument, since if the quanta $q_{a, h}$ are too small, the interaction of the 3 -form with the membranes would be weaker than the gravitation of the latter, i.e.

$$
m_{\text {axion }} \gtrsim \frac{T}{2 \pi f M_{p}} \simeq \frac{M^{3}}{2 \pi f M_{p}} .
$$


Combining it with the stability constraint $M^{2} \geq(0.5) \eta^{-1} m m_{H}$ one has a lower bound

$$
m_{\text {axion }} \gtrsim \frac{0.3}{2 \pi} \eta^{-3 / 2} \frac{\left(m_{H}^{2} m^{2}\right)^{3 / 4}}{f M_{p}}
$$

We thus see that there is a wide range of possible axion masses. Depending on the values of the axion periodicity $f$ and the Higgs mass UV cut-off $m$ one has

$$
4.7 \eta^{-3 / 2} 10^{-3} \mathrm{eV}\left(\frac{10^{10} \mathrm{GeV}}{f}\right)\left(\frac{m}{10^{10} \mathrm{GeV}}\right)^{3 / 2} \lesssim m_{\text {axion }} \lesssim \eta^{-1} 10^{-6} \mathrm{GeV}\left(\frac{10^{10} \mathrm{GeV}}{f}\right)
$$

where we have highlighted the values for $f \simeq m \simeq 10^{10} \mathrm{GeV}$. Note that as long as the constraint (3.24) is fulfilled, both upper and lower limits are consistent. As we can see, a very wide range of values of the hierarxion mass are consistent with the generation of a SM landscape. Thus for $\eta \simeq 1$ and $f \simeq m \simeq 10^{10} \mathrm{GeV}$ one has $10^{-3} \mathrm{eV} \lesssim m_{\text {axion }} \lesssim 10^{3} \mathrm{eV}$. However, if the Hierarxion does not couple directly to gauge bosons and $f \simeq 10^{2} \mathrm{GeV}$, the hieraxion could be as heavy as hundreds of GeV. In this case it could mix with the ordinary Higgs. Furthermore, if the hieraxion-Higgs coupling $\eta$ is small, the Hierarxion may have even larger masses. For example, if $\eta \simeq 10^{-16}$, one could heav a Hierarxion mass as large as $10^{10} \mathrm{GeV}$.

This Hierarxion can give rise to interesting phenomenology which will obviously depend on the values of the mass and $f$, and also on the existence of additional couplings to the SM beyond the necessary coupling to the Higgs. Here we limit ourselves to a preliminary discussion and leave a detail discussion for forthcoming work. In particular a light Hierarxion could be a dark matter component. CMB Planck results already constrain in an important manner the contribution to dark matter from ultralight axions. In the region $10^{-32} \mathrm{eV} \leq m_{\text {axion }} \leq 10^{-26} \mathrm{eV}$ the axion contribution to dark matter is less than a few per cent (see e.g. [55] and references therein). On the other hand for axion masses above $10^{-23} \mathrm{eV}$ an ultralight Hierarxion could constitute most of dark matter.

The Hierarxion needs not couple to gluons or photons, but if it does, it could perhaps be identified with the QCD axion. However the axion potential discussed above can overwhelm the standard non-perturbative QCD axion potential and spoil the solution to the strong $\mathrm{CP}$ problem and render $\theta \simeq 1$. To avoid that, one imposes the constraint

$$
\left(q m^{2} / \eta\right) \lesssim \theta_{\mathrm{QCD}} \Lambda_{\mathrm{QCD}}^{4}
$$

where $q$ stands for $q_{a, h}$ and the $\theta_{\mathrm{QCD}}$ is constrained to be $\theta_{\mathrm{QCD}} \leq 10^{-10}$. This means

$$
q \lesssim \eta \theta_{\mathrm{QCD}} \frac{\Lambda_{\mathrm{QCD}}^{4}}{m^{2}},
$$

and the EW scale fine-tuning, which is of order $q$, would be much finer than just $q \simeq M_{H}^{2}$. Such small value for $q$ however implies, if the WGC applies, that membranes should have a tension

$$
T \leq q M_{p} \lesssim \eta \theta_{\mathrm{QCD}} \frac{M_{p} \Lambda_{\mathrm{QCD}}^{4}}{m^{2}} \lesssim \eta\left(\frac{100 \mathrm{GeV}}{m}\right)^{2} \mathrm{GeV}^{3}
$$


This tension is typically very small, well below the EW scale, and hence we should have observed the new physics associated to the membranes. Another possible objection to such small quanta $q$ is that membrane nucleation could destabilize the minima through tunneling, as discussed above. It is easy to convince oneself using the equations above that the tunneling rate $B$ would easily be much bigger than one if the scalar mass cut-off obeys

$$
m^{2} \lesssim \eta \theta_{\mathrm{QCD}}^{1 / 4} \Lambda_{\mathrm{QCD}} M_{p}
$$

which is easily obeyed for $m \leq \eta^{1 / 2} 10^{7} \mathrm{GeV}$.

Very massive Hierarxions are also possible. Looking to the upper limit in equation (3.29) we see that one can have Hierarxions with mass of order

$$
m_{\text {axion }} \simeq f \simeq 10^{2} \mathrm{GeV}
$$

Such a Megaxion could be directly detectable at LHC if it couples to QCD and photons, since it could lead to di-photon events at an invariant mass in the region of several hundred $\mathrm{GeV} .{ }^{4}$ In this case the axion could have a non-negligible mixing with the SM Higgs which could also lead to constraints in the axion-Higgs system. Note that in this case, having $f$ of order a few hundred $\mathrm{GeV}$ implies that some new physics should appear not much above one $\mathrm{TeV}$ in order to restore perturbative unitarity, which would be violated by the coupling of the Hierarxion to gluons at high energies.

The Hieraxion may also be superheavy, with a mass of order the intermediate scale. This is possible if it is very weakly coupled to the Higgs sector. For example if $\eta \simeq 10^{-16}$ one can have a mass of order $10^{10} \mathrm{GeV}$ for $f \simeq 10^{10} \mathrm{GeV}$. Superheavy masses for the Hierarxion are also natural in the context of supersymmetry.

Let us finally emphasize that the example above, with a linear coupling to the axion $\eta \phi F_{4}$ is minimal, but is not the only possibility. One could also consider e.g. quadratic couplings of the form $F_{4}^{2}|H|^{2} / M_{\mathrm{UV}}^{2}$, with $M_{\mathrm{UV}}$ some ultraviolet scale. This structure appears naturally in the SUSY case which we describe below.

\section{A MSSM landscape}

It is interesting to explore whether analogous landscapes could be constructed within $N=1$ SUSY models like e.g. the MSSM. It sounds a bit redundant to introduce SUSY in theories in which the hierarchy problem is solved via a landscape of Higgss masses. However this may be interesting because of several reasons. For example, there are SUSY models in the literature, like Split SUSY [59, 60] or Large Scale SUSY [61] in which the scale of SUSY breaking is very large, of order $10^{5}-10^{11} \mathrm{GeV}$ and the Higgs mass is small by fine-tuning. For those models a landscape of soft terms guaranteeing the possibility of a sufficiently light Higgs would be useful. Furthermore one can also consider this type of fine-tuning in order to understand or motivate the so called "little hierarchy problem".

\footnotetext{
${ }^{4}$ Models with that structure were suggested as arising in string compactifications with a string scale in the $\mathrm{TeV}$ scale [56-58]. In this case the axion is a Ramond-Ramond closed string pseudoscalar.
} 
For a SUSY version of a landscape we should start by asking whether there are SUSY multiplets incorporating 3-forms of the type discussed above. A hint to that is noticing that the Minkowski 4-forms do not propagate, but rather behave like auxiliary fields. So it is natural to think that the Minkowski 4-forms could appear as auxiliary fields of some known SUSY multiplets. Indeed, there are SUSY chiral multiplets in which the usual complex auxiliary field are totally or partially replaced by 4 -forms [52, 62-72]. Still these multiplets have not been much studied in the literature.

Interestingly enough it has been recently shown $[25,73]$ that this kind of supergravity and supersymmetry multiplets are those which naturally appear in Type II string compatifications in the presence of fluxes. In string compactifications the geometric moduli and the dilaton come along with axion-like scalar fields. One can show that the dependence of the effective action on the axions comes always through Minkowski 4-forms, very much like in the non-SUSY example above. In the case of Type IIA and Type IIB orientifolds the effective actions contain 4-forms associated to the moduli and complex dilaton and the scalar potential dependence of the axions appears as a sum of squared 4-forms. These 4-forms may be identified as auxiliary fields of $N=1$ multiplets.

Note that having 4-forms as auxiliary fields is not purely academic since there are a number of physical differences compared to a standard $N=1$ sugra auxiliary field. In particular the associated 3-forms couple to membranes, which should be present in the theory. The membranes can nucleate inducing transitions between vacua with different value for the 4 -form. Furthermore the 4 -forms are in general quantized. In particular in string-theory the value of the 4 -forms is dual to the (quantized) value of internal fluxes. This means that there is a quantized landscape of auxiliary fields in the effective field theory, and transitions between different vacua can in principle proceed through membrane nucleation. (A somewhat related approach has been also recently considered in [74] involving in addition a nihilpotent multiplet and applying it to the cancellation of the cosmological constant.)

Let us consider a toy $N=1$ supergravity example with the required built-in discrete shift symmetries, consistent with having quantized 4 -forms as auxiliary fields. Take a 2 -field model with

$$
K=-2 \log \left(U+U^{*}\right)-3 \log \left(T+T^{*}\right), \quad W=e_{0}+i h_{0} U
$$

With $U=u+i b$ and $T=t+i \operatorname{Im}(T)$ the action will be invariant under the shift symmetry

$$
b \rightarrow b+n ; \quad e_{0} \rightarrow e_{0}+h_{0} n, \quad n \in \mathbf{Z}
$$

Consistency requires $e_{0}$ to be quantized in units of $h_{0}$. In string theory models these numbers are in general integers (see e.g. [76] and references therein), corresponding to quanta of internal fluxes, and we assume so in what follows. This is a no-scale model and the associated potential may be obtained in the standard way yielding

$$
V=e^{K} G_{U \bar{U}}^{-1}\left|D_{U} W\right|^{2}=e^{K} 2\left|e_{0}-h_{0} b\right|^{2} .
$$

The potential is shift invariant and has minima at $b=e_{0} / h_{0}$ in Minkowski, and the rest of the fields are undetermined at this level. Supersymmetry is broken and the gravitino mass 
is given by

$$
m_{3 / 2}^{2}=\frac{h_{0}^{2}}{2 t^{3}}
$$

The standard $N=1$ auxiliary fields are given by

$$
F^{U}=e^{K / 2} 2 u\left(e_{0}-h_{0} b\right)=0 ; \quad F^{T}=-i e^{K / 2} 2 h_{0} u t \neq 0 .
$$

With $h_{0}$ quantized we have a landscape of values for the gravitino mass (for fixed $u, t$ ). Note that the scalar potential of this system may be understood in terms of a Minkowski 4-form with an action

$$
\mathcal{L}_{F}=-e^{-K} F_{4}^{2}+2 F_{4}\left(e_{0}-h_{0} b\right) .
$$

Upon application of the equations one obtains

$$
F_{4}=e^{K}\left(e_{0}-h_{0} b\right),
$$

and the scalar potential above is recovered. The $N=1$ auxiliary field for the $U$ field may be written in terms of this 4 -form

$$
F_{U}=2 u e^{-K / 2} F_{4} .
$$

Still, since its vev is proportional to $h_{0}$, which is quantized, the gravitino mass and soft terms are also quantized. This is an example of a $N=1$ sugra model consistent with a formulation in terms of 3-forms. Other examples obtained from TYpe IIA and TYpe IIB orientifold vacua may be found in [25].

We can consider now the addition of matter fields like e.g. a MSSM Higgs sector $H_{u, d}$ and use the above toy model as a "hidden sector" for it. If e.g. the Higgs fields had minimal canonical kinetic terms we will get for the Higgs mass (see e.g. [75]):

$$
m_{H_{u}}^{2}=m_{H_{d}}^{2}=m_{3 / 2}^{2}=\frac{h_{0}^{2} M_{s}^{4}}{2 M_{p}^{2} t^{3}}
$$

where we have re-inserted the relevant Planck and string mass factors which we were skipping up to now. For values of $h_{0} \simeq\left(10^{10} \mathrm{GeV}\right)^{2}$, we will have qualitatively

$$
m_{H} \simeq h_{0} \times 10^{2} \mathrm{GeV}\left(\frac{M_{s}^{2}}{10^{20} \mathrm{GeV}^{2}}\right) .
$$

Given that $h_{0}$ is quantized, the Higgs masses will scan in a landscape. This model is a "toy" since the rest of the scalar fields are undetermined, but that is inessential to the point we want to make, that there will be in general a landscape of Higgs masses if the auxiliary fields relevant of the hidden sector contain quantized 4-forms, as indicated by string theory.

In general the full scalar potential in a fully realistic MSSM depends on a variety of soft terms plus a $\mu_{0}$-term for the Higgs. At the end of the day, assuming for simplicity flavour independence and universality, the mass of the weak scale gauge bosons can be written as an expansion in terms of soft-terms

$$
M_{Z^{0}}^{2}=c_{1} M^{2}+c_{2} m^{2}+c_{3}|A|^{2}+c_{4}\left|\mu_{0}\right|^{2}+c_{5} M A+c_{6} \mu_{0} M+c_{7} \mu_{0} A \ldots
$$


where $c_{i}=c_{i}\left(y_{t}, g_{i}\right)$ are coefficients depending only on the gauge and Yukawa couplings and including all the running between the UV scale and the EW scale. Here, in a standard notation, $M$ is a universal gaugino mass, $m$ the soft scalar masses, $A$ is the trilinear soft coupling and $\mu_{0}$ is the SUSY Higgs mass. In one such more complete setting all these soft terms $\mathcal{M}_{\text {soft }}^{i}=m, M, A, B, \mu_{0}, \ldots$ will be quantized

$$
\mathcal{M}_{\mathrm{soft}}^{i}=n_{i} \frac{h_{0}^{i}}{M_{p}}, \quad n_{i} \in \mathbf{Z}
$$

where the $n_{i}$ of different soft terms need not be directly correlated, and the $h_{0}^{i}$ are of the same order. Thus we would have a grid of soft terms, with most of the points not giving appropriate EW symmetry breaking, but with some points consistent with correct EW breaking, with Higgs vevs consistent with anthropic considerations.

This built-in structure could perhaps explain the little hierarchy problem. Indeed, it could be that soft terms could be above a few $\mathrm{TeV}$, with squark and gluinos perhaps above LHC reach. But for particular choices of the integers $n_{i}$, cancellations could take place allowing for correct EW symmetry breaking with an apparently fine-tuned choice of SUSY parameters.

Let us comment about the connection between this SUSY landscape and the nonSUSY case considered in the previous section. In fact in the SUSY case, due to gravity mediation, the coupling of the 4-forms to the Higgs mass is quadratic and Planck supresed, rather than linear. One indeed has couplings of the form $F_{4}^{2}|H|^{2} / M_{p}^{2}$, rather than $\eta F|H|^{2}$. One gets a mass of order the EW scale for the axion in both cases if $\eta \simeq 10^{-8}$. There is also a difference in the kind of landscape achieved. In the non-SUSY case there is a delicate cancellation between the UV mass $m^{2}$ of the Higgs and the contribution of the 4-forms $f_{0}^{h}, f_{0}^{a}$ as in eq. (3.12). If $m^{2}$ is very large one needs $q_{a, h} \ll f_{a, h}$. On the other hand in the above SUSY landscape the value of the 4-forms (or auxiliary fields) is very large, naturally (although not necessarily) of order $10^{20} \mathrm{GeV}^{2}$, and the fine-tuning is naturally small, $\simeq F / M_{p} \simeq 100 \mathrm{GeV}$ because of gravity mediation.

\section{The SUSY breaking scale, the string scale and the Weak Gravity Con- jecture}

In this section we depart from the issue of the generation of a Higgs mass landscape and adopt a more general view, this time within string theory. We would like to argue that in large classes of string compactifications (see e.g. [76]), the WGC suggests that there is a lower bound on the SUSY breaking scale $m_{3 / 2}$, depending on the string scale $M_{s}$. Roughly the lower bound is given by

$$
m_{3 / 2} \gtrsim \frac{M_{s}^{2}}{M_{p}}
$$

This bound has a number of loopholes and should only be considered as a common feature in certain classes of compactifications. Still, since it would have important implications, we think it would be worth studying how general it is. 
Let us summarize the underlying idea. In the above $N=1$ SUSY examples the gravitino and soft-term masses scale like a quantized parameter $h_{0}$, which is an integer in units of some fundamental scale (i.e. $M_{s}^{2}$, with $M_{s}$ the string scale). In these models with quantized 4 -forms there are membranes coupling to the 3 -forms. The charge of these membranes, of mass dimension two, will be proportional to $h_{0}$ or, reintroducing the axion period $f$, to $\mu f$, with $\mu$ the axion mass term. Now, as in the examples above, the WGC as applied to 3 -forms and membranes give an upper bound on the tension $T$ of the membranes coupling to a 3 -form with charge $q[24]$

$$
\frac{T}{M_{p}} \leq 2 \pi q
$$

i.e., the strength of the 3 -form coupling must be bigger than the gravitational coupling of the membrane. Applying these conditions to the axions $\phi_{\alpha}$ of some consistent string compactification one expects for all of them

$$
\frac{T^{\alpha}}{f_{\alpha} M_{p}} \leq 2 \pi \mu_{\alpha}
$$

as long as they couple to a massive 3 -form. This is interesting because it is telling us that all these axions cannot be arbitrarily light, since their mass corresponds to the coupling of 3 -forms to membranes, which cannot be small in order not to violate the WGC. This should be preserved in any consitent compactification.

In principle one can go case by case and test in specific string compactifications whether the spectra of axion masses respects the bounds (5.3). That may give relevant constraints on specific moduli fixing vacua and provide explicit tests of the WGC. However one can draw some general expectations from the given structure. In particular, there are general classes of models in which axion masses are directly related to the SUSY-breaking scale. Those are models in which the Goldstino multiplet contains a monodromy axion. In that case the mass of the axion is of the order of the gravitino mass and hence the bound applies not only to the axion but to the gravitino itself, i.e.

$$
m_{3 / 2} \simeq m_{\alpha} \geq \frac{T_{\alpha}}{f_{\alpha} M_{p}}
$$

Models in which the Goldstino contains a monodromy axion include Type IIA or Type IIB orientifolds with all moduli fixed by RR, NS and eventually additional geometric or nongeometric fluxes, see $[87,88]$ and references therein. In these models SUSY is broken by the auxiliary fields of either Kahler, complex structure and/or complex dilaton. Thus some linear combination of axions will be SUSY partners of the Goldstino/gravitino, and the bound above would apply. More generally, in typical string compactifications with broken SUSY and stabilized moduli, either the Kahler, complex structure or complex dilaton auxiliary fields tipically dominate SUSY breaking. In these cases some linear combination of the axions in the moduli will be a SUSY partner of the Goldstino/gravitino. So at least the mass of that particular linear combination will be of order the gravitino mass, $m_{a} \simeq m_{3 / 2}$.

The bounds depend also on the membrane tensions and the periodicities. Concerning the axion periodicities $f_{\alpha}$, in string compactifications like these one typically has $f_{\alpha} \simeq$ 
$M_{s}$, although values as large as $M_{p}$ or slightly below $M_{s}$ are also possible, depending on volume factors. Concerning the tensions of the RR membranes, they are in principle proportional to the volume wrapped by the higher dimensional D-branes or NS-branes yielding membranes upon compactification. One may argue that one can make the tensions arbitrarily small by making the cycles of the volumes arbitrarily small, which would make the bounds (5.3) weaker. However we would have to do that simultaneously with all the 3forms and membranes, which sounds artificial. Furthermore, as emphasized already in [16] , although the classical tensions can be vanishingly small, the effective tensions are only slightly smaller than $M_{s}^{3}$. This is because the Weil-Peterson metric in e.g. a conifold cycle scales logarithmically with the blowing-up mode [16]. In any event, let us evaluate the bounds by setting the tensions $T_{\alpha} \simeq M_{s}^{3}$. One gets

$$
m_{3 / 2} \simeq m_{\alpha} \geq \frac{T_{\alpha}}{f_{\alpha} M_{p}} \simeq \frac{M_{s}^{2}}{M_{p}}
$$

as advertised. We are also assuming here that $g_{s} \simeq 1$, as happens in semirealistic compactifications in which one adjusts the gauge couplings to the observed values. Thus we see that the scale of SUSY breaking cannot be arbitrarily low. That would imply the existence of interactions of some 3 -form with membranes with strength weaker than that of gravity.

The above bound, if true, would have important phenomenological implications. In this connection there are a couple of situations of particular phenomenological interest:

- Intermediate scale SUSY breaking. In this case SUSY is broken at $m_{3 / 2} \simeq 10^{12} \mathrm{GeV}$ and $M_{s} \simeq 10^{15} \mathrm{GeV}$, consistent with the bound. The spectrum below $m_{3 / 2}$ is that of the minimal SM. This is interesting because it is known that, if one extrapolates the SM Higgs potential corresponding to a $126 \mathrm{GeV}$ Higgs at high energies, the potential develops an instability at around $10^{10} \mathrm{GeV}$ [77]. If SUSY is restored above $10^{10} \mathrm{GeV}$ such instability disappears. This situation with an intermediate SUSY scale $M_{S S}$ has also been recently discussed both in the context of the observed SM Higgs mass [78, 79] as well as in MSSM Higgs inflation [80-82]. In this situation no SUSY particles would be observed at LHC.

- $S U S Y$ at a TeV. In this case one can have $m_{3 / 2} \simeq \mathrm{TeV}$ with an intermediate string scale $M_{s} \simeq 10^{10}$, also consistent with the bound. This is the case discussed in the previous section in the context of a MSSM landscape. In this case SUSY particles could perhaps be observed at LHC but standard unification of coupling constants is lost. The case with an intermediate string scale has been considered from different considerations in the literature (see e.g. [83]). Note in particular that it was found in the context of Type IIB orientifolds with fluxes that SUSY breaking soft terms are obtained for the MSSM scalars living on D7-branes which scale precisely in the same way, with [84]

$$
m_{\mathrm{soft}} \simeq \frac{f M_{s}^{2}}{M_{p}}
$$


and $f$ parametrizing the local fluxes on the brane positions. This soft terms would be consistent with the bound on SUSY breaking discussed above. ${ }^{5}$

On the other hand a big dessert scenario with the GUT/String scale at $M_{s} \simeq 10^{16} \mathrm{GeV}$ and low energy SUSY at a TeV, leading to succesful gauge coupling unification would be inconsistent with such a bound.

Let us close this section by noting that axions may get also a potential from instanton effects rather than directly from fluxes. This happens for example in Type IIB compactifications with standard NS and RR fluxes. The latter only induce monodromy to the complex structure and dilaton fields, but not to the axions in the Kahler multiplets. In the presence of gaugino condensation the role of the 3-forms is played by the composite CS 3form of the condensing gauge group, see [17-20,49]. In this case the associated membrane tension is of order $T \simeq \Lambda^{3}$, with $\Lambda$ the condensate scale, and the bound above constraint $\Lambda$ instead of the string scale. A similar effect may be described in terms of a warping throat. ${ }^{6}$ The origin of the composite 3 -forms associated to non-gauge string instantons has been recently worked out in [49].

\section{Comments and conclusions}

In this paper we have studied how to generate a landscape of Higgs masses in order to address the gauge hierachy problem. Although anthropic considerations based on the viability of complex nuclei constrain the Higgs vev to be close to the observed value, we still need to have theories in which a landscape of Higgss masses, including viable ones, appear. This is what we tried to address in the present paper.

We put forward a general mechanism in which the landscape properties of an axion-3form system is transmitted to the Higgs sector of the SM or the MSSM. Indeed, the 4-form field strengths associated to 3 -forms are assumed to be quantized, as e.g. happens in string theory. On the other hand there is an axion-like field which 1 ) gives a mass to the 3 -form and 2) couples to the Higgs field. Then the quantization properties of the axion/3-form system is transmitted to the Higgs sector via either a direct renormalizable coupling (as in a non-SUSY example discussed above) or mediated by gravity, as in the SUSY examples discussed in the previous section.

In the non-SUSY examples the mechanism suggests the existence of axion-like scalars with very weak couplings to the SM sector. Arguments based on the Weak Gravity Conjecture suggests masses for this Hierarxion not much below $10^{-3} \mathrm{eV}$, although the possible range of values is very large. In order to generate the landscape it is not needed that this axion couples directly to the QCD or photon field strengths, as ordinary axions do. On the other hand it can contribute to dark matter, although the chances to detect this axion with standard techniques is model dependent. One can contemplate the possibility of this axion to be identified with an ordinary PQ axion, but the fact that it couples to the Higgs

\footnotetext{
${ }^{5}$ Note however that the masses of SM SUSY partners could present further supression compared to the value of $m_{3 / 2}$. As an example, in the Large Volume Scenario $[85,86]$ a sequestering effect can make $m_{\text {soft }} \ll m_{3 / 2}$.

${ }^{6}$ We thank the referee for emphasizing this point to us.
} 
sector makes difficult to achieve that goal, since its potential dominates over the standard instanton-induced potential. It would be interesting to study different models in which different detection opportunities could be present.

In the SUSY examples, the axion/3-form system appears as part of the auxiliary fields involved in gravity mediation models. The presence of Minkowski 4-forms behaving as auxiliary fields of $N=1$ supergravity has been recently shown to be a general property in string theory. In this case the philosophy is a bit different since the most natural situation is one in which the string scale is identified with an intermediate scale $M_{s} \simeq f \simeq 10^{10} \mathrm{GeV}$. The SUSY breaking soft terms are then of order $\mathcal{M}_{\text {soft }} \simeq F_{4} / M_{p} \simeq M_{s}^{2} / M_{p}$ and scan in a landscape, with values of order the EW scale. There is a landscape of soft terms which could perhaps provide a qualitative understanding of the SUSY fine-tuning implied by LHC results.

The fact that in the SUSY case the interaction of 4-forms with the Higgs field is Planck supresed yields a small EW scale in a natural way. This is to be compared to the non-SUSY case in which the scale $\eta q$ is assumed to be smaller than $M_{W}$ ab initio. On the other hand the non-SUSY version predicts the existence of an axion-like particle which could lead to interesting phenomenology.

In both cases, SUSY and non-SUSY, the Weak Gravity Conjecture, as applied to 3forms, suggests that there is a scale of new physics well below the Planck mass. Indeed, we saw that in the non-SUSY class of models such an scale of order $\eta^{-1 / 3} 10^{8} \mathrm{GeV}$ or below should exist. In the SUSY case the string scale should typically be of order of the intermediate scale $10^{10} \mathrm{GeV}$ or so, to generate a landscape.

More generally, one can argue that in large classes of string compactifications with fluxes the WGC suggests a lower bound on the SUSY breaking scale with $m_{3 / 2} \gtrsim M_{s}^{2} / M_{p}$. This applies in particular to models in which the Goldstino multiplet contains a monodromy axion, but it could be more general. Although, admittedly, there are a number of loopholes in such a bound, it would be interesting to test it in specific compactifications.

Note that in here we have not addressed the problem of the cosmological constant. We are tacitaly assuming that there is a different mechanism, like the Bousso-Polchinski (BP) mechanism [15] which addresses this issue. Note that the mechanism discussed here is not of the BP type, in which delicate cancellations of a large (on the hundreds) multiplicity of 4 -forms with large values, allows for the fine-tuning of the cosmological constant. One could think of the possibility of addressing the issue of the c.c. in a way analogous to the mechanism discussed in the present paper. However the scale of the cosmological constant is so small (of order $10^{-48} \mathrm{GeV}^{4}$ ) that a threshold of new-physics associated to the required axion/3-form system should have been already detected experimentally. We think on the other hand that a landscape for the EW sector appears more naturally in the context of axion/3-form systems as here described.

\section{Acknowledgments}

We thank F. Marchesano, V. Martín-Lozano, M. Montero, A. Uranga and I. Valenzuela for useful discussions. This work has been supported by the ERC Advanced Grant SPLE under contract ERC-2012-ADG-20120216-320421, by the grant FPA2012-32828 from the 
MINECO, and the grant SEV-2012-0249 of the "Centro de Excelencia Severo Ocho" Programme. The work of A. Herráez is supported by a FPU fellowship from MINECO.

Open Access. This article is distributed under the terms of the Creative Commons Attribution License (CC-BY 4.0), which permits any use, distribution and reproduction in any medium, provided the original author(s) and source are credited.

\section{References}

[1] S. Weinberg, The cosmological constant problem, Rev. Mod. Phys. 61 (1989) 1 [InSPIRE].

[2] T. Damour and J.F. Donoghue, Constraints on the variability of quark masses from nuclear binding, Phys. Rev. D 78 (2008) 014014 [arXiv: 0712.2968] [INSPIRE].

[3] J.F. Donoghue, K. Dutta, A. Ross and M. Tegmark, Likely values of the Higgs vev, Phys. Rev. D 81 (2010) 073003 [arXiv: 0903.1024] [INSPIRE].

[4] L.J. Hall, D. Pinner and J.T. Ruderman, The weak scale from BBN, JHEP 12 (2014) 134 [arXiv: 1409.0551] [INSPIRE].

[5] U.-G. Meißner, Anthropic considerations in nuclear physics, Sci. Bull. 60 (2015) 43 [arXiv: 1409.2959] [INSPIRE].

[6] J.F. Donoghue, The multiverse and particle physics, Ann. Rev. Nucl. Part. Sci. 66 (2016) 1 [arXiv: 1601.05136] [INSPIRE].

[7] N. Arkani-Hamed, S. Dimopoulos and S. Kachru, Predictive landscapes and new physics at a TeV, hep-th/0501082 [INSPIRE].

[8] M.J. Duff and P. van Nieuwenhuizen, Quantum inequivalence of different field representations, Phys. Lett. B 94 (1980) 179 [INSPIRE].

[9] S.W. Hawking, The cosmological constant is probably zero, Phys. Lett. B 134 (1984) 403 [INSPIRE].

[10] M.J. Duff, The cosmological constant is possibly zero, but the proof is probably wrong, Phys. Lett. B 226 (1989) 36 [inSPIRE].

[11] Z.C. Wu, The cosmological constant is probably zero and a proof is possibly right, Phys. Lett. B 659 (2008) 891 [arXiv:0709.3314] [INSPIRE].

[12] M.J. Duncan and L.G. Jensen, Four forms and the vanishing of the cosmological constant, Nucl. Phys. B 336 (1990) 100 [inSPIRE].

[13] J.D. Brown and C. Teitelboim, Neutralization of the cosmological constant by membrane creation, Nucl. Phys. B 297 (1988) 787 [INSPIRE].

[14] J.D. Brown and C. Teitelboim, Dynamical neutralization of the cosmological constant, Phys. Lett. B 195 (1987) 177 [inSPIRE].

[15] R. Bousso and J. Polchinski, Quantization of four form fluxes and dynamical neutralization of the cosmological constant, JHEP 06 (2000) 006 [hep-th/0004134] [INSPIRE].

[16] J.L. Feng, J. March-Russell, S. Sethi and F. Wilczek, Saltatory relaxation of the cosmological constant, Nucl. Phys. B 602 (2001) 307 [hep-th/0005276] [INSPIRE].

[17] G. Dvali, Large hierarchies from attractor vacua, Phys. Rev. D 74 (2006) 025018 [hep-th/0410286] [INSPIRE]. 
[18] G. Dvali, Three-form gauging of axion symmetries and gravity, hep-th/0507215 [INSPIRE].

[19] G. Dvali, A vacuum accumulation solution to the strong CP problem, Phys. Rev. D 74 (2006) 025019 [hep-th/0510053] [INSPIRE].

[20] G. Dvali, S. Folkerts and A. Franca, How neutrino protects the axion, Phys. Rev. D 89 (2014) 105025 [arXiv:1312.7273] [INSPIRE].

[21] N. Kaloper and L. Sorbo, A natural framework for chaotic inflation, Phys. Rev. Lett. 102 (2009) 121301 [arXiv:0811.1989] [INSPIRE].

[22] N. Kaloper, A. Lawrence and L. Sorbo, An ignoble approach to large field inflation, JCAP 03 (2011) 023 [arXiv:1101.0026] [INSPIRE].

[23] F. Marchesano, G. Shiu and A.M. Uranga, F-term axion monodromy inflation, JHEP 09 (2014) 184 [arXiv: 1404.3040] [INSPIRE].

[24] L.E. Ibáñez, M. Montero, A. Uranga and I. Valenzuela, Relaxion monodromy and the weak gravity conjecture, JHEP 04 (2016) 020 [arXiv: 1512.00025] [INSPIRE].

[25] S. Bielleman, L.E. Ibáñez and I. Valenzuela, Minkowski 3-forms, flux string vacua, axion stability and naturalness, JHEP 12 (2015) 119 [arXiv:1507.06793] [INSPIRE].

[26] C. Vafa, The string landscape and the swampland, hep-th/0509212 [INSPIRE].

[27] N. Arkani-Hamed, L. Motl, A. Nicolis and C. Vafa, The string landscape, black holes and gravity as the weakest force, JHEP 06 (2007) 060 [hep-th/0601001] [INSPIRE].

[28] H. Ooguri and C. Vafa, On the geometry of the string landscape and the swampland, Nucl. Phys. B 766 (2007) 21 [hep-th/0605264] [inSPIRE].

[29] T. Rudelius, Constraints on axion inflation from the weak gravity conjecture, JCAP 09 (2015) 020 [arXiv: 1503.00795] [INSPIRE].

[30] M. Montero, A.M. Uranga and I. Valenzuela, Transplanckian axions!?, JHEP 08 (2015) 032 [arXiv: 1503.03886] [INSPIRE].

[31] J. Brown, W. Cottrell, G. Shiu and P. Soler, Fencing in the swampland: quantum gravity constraints on large field inflation, JHEP 10 (2015) 023 [arXiv: 1503.04783] [INSPIRE].

[32] J. Brown, W. Cottrell, G. Shiu and P. Soler, On axionic field ranges, loopholes and the weak gravity conjecture, JHEP 04 (2016) 017 [arXiv: 1504.00659] [INSPIRE].

[33] B. Heidenreich, M. Reece and T. Rudelius, Weak gravity strongly constrains large-field axion inflation, JHEP 12 (2015) 108 [arXiv:1506.03447] [INSPIRE].

[34] C. Cheung and G.N. Remmen, Naturalness and the weak gravity conjecture, Phys. Rev. Lett. 113 (2014) 051601 [arXiv: 1402.2287] [INSPIRE].

[35] A. de la Fuente, P. Saraswat and R. Sundrum, Natural inflation and quantum gravity, Phys. Rev. Lett. 114 (2015) 151303 [arXiv: 1412.3457] [INSPIRE].

[36] A. Hebecker, P. Mangat, F. Rompineve and L.T. Witkowski, Winding out of the swamp: evading the weak gravity conjecture with F-term winding inflation?, Phys. Lett. B 748 (2015) 455 [arXiv: 1503.07912] [inSPIRE].

[37] T.C. Bachlechner, C. Long and L. McAllister, Planckian axions and the weak gravity conjecture, JHEP 01 (2016) 091 [arXiv:1503. 07853] [INSPIRE].

[38] T. Rudelius, On the possibility of large axion moduli spaces, JCAP 04 (2015) 049 [arXiv:1409.5793] [INSPIRE]. 
[39] D. Junghans, Large-field inflation with multiple axions and the weak gravity conjecture, JHEP 02 (2016) 128 [arXiv: 1504.03566] [INSPIRE].

[40] K. Kooner, S. Parameswaran and I. Zavala, Warping the weak gravity conjecture, Phys. Lett. B 759 (2016) 402 [arXiv: 1509.07049] [InSPIRE].

[41] D. Harlow, Wormholes, emergent gauge fields and the weak gravity conjecture, JHEP 01 (2016) 122 [arXiv:1510.07911] [INSPIRE].

[42] A. Hebecker, F. Rompineve and A. Westphal, Axion monodromy and the weak gravity conjecture, JHEP 04 (2016) 157 [arXiv: 1512.03768] [INSPIRE].

[43] M. Montero, G. Shiu and P. Soler, The weak gravity conjecture in three dimensions, JHEP 10 (2016) 159 [arXiv:1606.08438] [INSPIRE].

[44] H. Ooguri and C. Vafa, Non-supersymmetric AdS and the swampland, arXiv:1610.01533 [INSPIRE].

[45] B. Freivogel and M. Kleban, Vacua Morghulis, arXiv:1610.04564 [INSPIRE].

[46] P. Saraswat, Weak gravity conjecture and effective field theory, Phys. Rev. D 95 (2017) 025013 [arXiv:1608.06951] [INSPIRE].

[47] D. Klaewer and E. Palti, Super-Planckian spatial field variations and quantum gravity, JHEP 01 (2017) 088 [arXiv:1610.00010] [INSPIRE].

[48] L. McAllister, P. Schwaller, G. Servant, J. Stout and A. Westphal, Runaway relaxion monodromy, arXiv:1610.05320 [INSPIRE].

[49] E. García-Valdecasas and A. Uranga, On the 3-form formulation of axion potentials from D-brane instantons, arXiv: 1605.08092 [INSPIRE].

[50] M. Berasaluce-González, P.G. Cámara, F. Marchesano and A.M. Uranga, $Z_{p}$ charged branes in flux compactifications, JHEP 04 (2013) 138 [arXiv:1211.5317] [INSPIRE].

[51] M. Berasaluce-González, G. Ramírez and A.M. Uranga, Antisymmetric tensor $Z_{p}$ gauge symmetries in field theory and string theory, JHEP 01 (2014) 059 [arXiv:1310.5582] [INSPIRE].

[52] E. Dudas, Three-form multiplet and inflation, JHEP 12 (2014) 014 [arXiv:1407.5688] [INSPIRE].

[53] P.W. Graham, D.E. Kaplan and S. Rajendran, Cosmological relaxation of the electroweak scale, Phys. Rev. Lett. 115 (2015) 221801 [arXiv:1504.07551] [INSPIRE].

[54] S.R. Coleman and F. De Luccia, Gravitational effects on and of vacuum decay, Phys. Rev. D 21 (1980) 3305 [INSPIRE].

[55] D.J.E. Marsh, Axion cosmology, Phys. Rept. 643 (2016) 1 [arXiv:1510.07633] [INSPIRE].

[56] L.E. Ibáñez and V. Martín-Lozano, A megaxion at $750 \mathrm{GeV}$ as a first hint of low scale string theory, JHEP 07 (2016) 021 [arXiv:1512.08777] [INSPIRE].

[57] M. Cvetič, J. Halverson and P. Langacker, String consistency, heavy exotics and the $750 \mathrm{GeV}$ diphoton excess at the LHC, Fortschr. Phys. 64 (2016) 748 [arXiv:1512.07622] [INSPIRE].

[58] L.A. Anchordoqui, I. Antoniadis, H. Goldberg, X. Huang, D. Lüst and T.R. Taylor, $750 \mathrm{GeV}$ diphotons from closed string states, Phys. Lett. B 755 (2016) 312 [arXiv:1512.08502] [INSPIRE]. 
[59] N. Arkani-Hamed and S. Dimopoulos, Supersymmetric unification without low energy supersymmetry and signatures for fine-tuning at the LHC, JHEP 06 (2005) 073 [hep-th/0405159] [INSPIRE].

[60] G.F. Giudice and A. Romanino, Split supersymmetry, Nucl. Phys. B 699 (2004) 65 [Erratum ibid. B 706 (2005) 487] [hep-ph/0406088] [INSPIRE].

[61] L.J. Hall and Y. Nomura, A finely-predicted Higgs boson mass from a finely-tuned weak scale, JHEP 03 (2010) 076 [arXiv:0910.2235] [INSPIRE].

[62] S.J. Gates, Jr., Super p-form gauge superfields, Nucl. Phys. B 184 (1981) 381 [INSPIRE].

[63] S.J. Gates, Jr. and W. Siegel, Variant superfield representations, Nucl. Phys. B 187 (1981) 389 [inSPIRE].

[64] P. Binetruy, F. Pillon, G. Girardi and R. Grimm, The three form multiplet in supergravity, Nucl. Phys. B 477 (1996) 175 [hep-th/9603181] [inSPIRE].

[65] B.A. Ovrut and D. Waldram, Membranes and three form supergravity, Nucl. Phys. B 506 (1997) 236 [hep-th/9704045] [INSPIRE].

[66] P. Binetruy, G. Girardi and R. Grimm, Supergravity couplings: a geometric formulation, Phys. Rept. 343 (2001) 255 [hep-th/0005225] [INSPIRE].

[67] G. Girardi, R. Grimm, B. Labonne and J. Orloff, Correspondence between 3-form and non-minimal multiplet in supersymmetry, Eur. Phys. J. C 55 (2008) 95 [arXiv:0712.1923] [INSPIRE].

[68] B.B. Deo and S.J. Gates, Comments on nonminimal $N=1$ scalar multiplets, Nucl. Phys. B 254 (1985) 187 [InSPIRE].

[69] I.A. Bandos and C. Meliveo, Three form potential in (special) minimal supergravity superspace and supermembrane supercurrent, J. Phys. Conf. Ser. 343 (2012) 012012 [arXiv: 1107.3232] [INSPIRE].

[70] H. Nishino and S. Rajpoot, Alternative auxiliary fields for chiral multiplets, Phys. Rev. D 80 (2009) 127701 [INSPIRE].

[71] K. Groh, J. Louis and J. Sommerfeld, Duality and couplings of 3-form-multiplets in $N=1$ supersymmetry, JHEP 05 (2013) 001 [arXiv: 1212.4639] [INSPIRE].

[72] D.G. Cerdeño, A. Knauf and J. Louis, A note on effective $N=1$ super Yang-Mills theories versus lattice results, Eur. Phys. J. C 31 (2003) 415 [hep-th/0307198] [INSPIRE].

[73] F. Carta, F. Marchesano, W. Staessens and G. Zoccarato, Open string multi-branched and Kähler potentials, JHEP 09 (2016) 062 [arXiv: 1606.00508] [INSPIRE].

[74] F. Farakos, A. Kehagias, D. Racco and A. Riotto, Scanning of the supersymmetry breaking scale and the gravitino mass in supergravity, JHEP 06 (2016) 120 [arXiv:1605.07631] [INSPIRE].

[75] A. Brignole, L.E. Ibáñez and C. Muñoz, Soft supersymmetry breaking terms from supergravity and superstring models, Adv. Ser. Direct. High Energy Phys. 18 (1998) 125 [hep-ph/9707209] [INSPIRE].

[76] L.E. Ibáñez and A.M. Uranga, String theory and particle physics: an introduction to string phenomenology, Cambridge Univ. Pr., Cambridge U.K., (2012) [INSPIRE].

[77] G. Degrassi et al., Higgs mass and vacuum stability in the Standard Model at NNLO, JHEP 08 (2012) 098 [arXiv: 1205.6497] [inSPIRE]. 
[78] L.E. Ibáñez, F. Marchesano, D. Regalado and I. Valenzuela, The intermediate scale MSSM, the Higgs mass and F-theory unification, JHEP 07 (2012) 195 [arXiv:1206.2655] [INSPIRE].

[79] L.E. Ibáñez and I. Valenzuela, The Higgs mass as a signature of heavy SUSY, JHEP 05 (2013) 064 [arXiv:1301.5167] [INSPIRE].

[80] L.E. Ibáñez, F. Marchesano and I. Valenzuela, Higgs-otic inflation and string theory, JHEP 01 (2015) 128 [arXiv:1411.5380] [INSPIRE].

[81] S. Bielleman, L.E. Ibáñez, F.G. Pedro and I. Valenzuela, Multifield dynamics in Higgs-otic inflation, JHEP 01 (2016) 128 [arXiv:1505.00221] [INSPIRE].

[82] S. Bielleman, L.E. Ibáñez, F.G. Pedro, I. Valenzuela and C. Wieck, The DBI action, higher-derivative supergravity and flattening inflaton potentials, JHEP 05 (2016) 095 [arXiv: 1602.00699] [INSPIRE].

[83] C.P. Burgess, L.E. Ibáñez and F. Quevedo, Strings at the intermediate scale, or is the Fermi scale dual to the Planck scale?, Phys. Lett. B 447 (1999) 257 [hep-ph/9810535] [InSPIRE].

[84] P.G. Cámara, L.E. Ibáñez and A.M. Uranga, Flux-induced SUSY-breaking soft terms on D7-D3 brane systems, Nucl. Phys. B 708 (2005) 268 [hep-th/0408036] [InSPIRE].

[85] V. Balasubramanian, P. Berglund, J.P. Conlon and F. Quevedo, Systematics of moduli stabilisation in Calabi-Yau flux compactifications, JHEP 03 (2005) 007 [hep-th/0502058] [INSPIRE].

[86] J.P. Conlon, F. Quevedo and K. Suruliz, Large-volume flux compactifications: moduli spectrum and D3/D7 soft supersymmetry breaking, JHEP 08 (2005) 007 [hep-th/0505076] [INSPIRE].

[87] B. de Carlos, A. Guarino and J.M. Moreno, Complete classification of Minkowski vacua in generalised flux models, JHEP 02 (2010) 076 [arXiv:0911.2876] [INSPIRE].

[88] B. de Carlos, A. Guarino and J.M. Moreno, Flux moduli stabilisation, supergravity algebras and no-go theorems, JHEP 01 (2010) 012 [arXiv:0907.5580] [INSPIRE]. 